\title{
Brain White Matter Correlates of Creativity in Schizophrenia: A Diffusion Tensor Imaging Study
}

\author{
Agurne Sampedro ${ }^{1}$, Javier Peña ${ }^{1 *}$, Naroa Ibarretxe-Bilbao ${ }^{1}$, \\ Alberto Cabrera-Zubizarreta ${ }^{2}$, Pedro Sánchez ${ }^{3,4}$, Ainara Gómez-Gastiasoro ${ }^{1}$, \\ Nagore Iriarte-Yoller ${ }^{3}$, Cristóbal Pavón ${ }^{3}$ and Natalia Ojeda ${ }^{1}$
}

'Department of Methods and Experimental Psychology, Faculty of Psychology and Education, University of Deusto, Billbao, Spain, ${ }^{2}$ OSATEK, MR Unit, Hospital of Galdakao, Galdakao, Spain, ${ }^{3}$ Refractory Psychosis Unit, Hospital Psiquiátrico de Álava, Vitoria-Gasteiz, Spain, ${ }^{4}$ Department of Neuroscience, Psychiatry Section, Faculty of Medicine and Odontology, University of the Basque Country (UPV/EHU), Leioa, Spain

OPEN ACCESS

Edited by:

Monica Luciana,

University of Minnesota Twin Cities,

United States

Reviewed by:

Kang Ik Kevin Cho, Harvard Medical School,

United States

Zora Kikinis,

Harvard Medical School,

United States

*Correspondence: Javier Peña javier.pena@deusto.es

Specialty section: This article was submitted to Decision Neuroscience, a section of the journal

Frontiers in Neuroscience

Received: 17 January 2020

Accepted: 11 May 2020

Published: 23 June 2020

Citation:

Sampedro A, Peña J Ibarretxe-Bilbao $N$ Cabrera-Zubizarreta A, Sánchez $P$, Gómez-Gastiasoro A, Iriarte-Yoller N,

Pavón C and Ojeda N (2020) Brain White Matter Correlates of Creativity in Schizophrenia: A Diffusion Tensor Imaging Study. Front. Neurosci. 14:572. doi: 10.3389/fnins.2020.00572
The relationship between creativity and psychopathology has been a controversial research topic for decades. Specifically, it has been shown that people with schizophrenia have an impairment in creative performance. However, little is known about the brain correlates underlying this impairment. Therefore, the aim of this study was to analyze whole brain white matter (WM) correlates of several creativity dimensions in people with schizophrenia. Fifty-five patients with schizophrenia underwent diffusionweighted imaging on a $3 \mathrm{~T}$ magnetic resonance imaging machine as well as a clinical and a creativity assessment, including verbal and figural creativity measures. Tractbased spatial statistic, implemented in FMRIB Software Library (FSL), was used to assess whole brain WM correlates with different creativity dimensions, controlling for sex, age, premorbid IQ, and medication. Mean fractional anisotropy (FA) in frontal, temporal, subcortical, brain stem, and interhemispheric regions correlated positively with figural originality. The most significant clusters included the right corticospinal tract (cerebral peduncle part) and the right body of the corpus callosum. Verbal creativity did not show any significant correlation. As a whole, these findings suggest that widespread WM integrity is involved in creative performance of patients with schizophrenia. Many of these areas have also been related to creativity in healthy people. In addition, some of these regions have shown to be particularly impaired in schizophrenia, suggesting that these WM alterations could be underlying the worse creative performance found in this pathology.

Keywords: creativity, schizophrenia, white matter, divergent thinking, psychosis, fractional anisotropy

\section{INTRODUCTION}

The relationship between creativity and schizophrenia has been a research topic of interest for centuries (Thys et al., 2013) since it was considered a key component for daily life problem solving (Plucker et al., 2015), and it seemed to have an impact on school, academic, and job performance (Rindermann and Neubauer, 2004). Although there are single famous cases of highly creative

Abbreviations: FA, fractional anisotropy; PANSS, Positive and Negative Syndrome Scale; WM, white matter. 
people with a possible diagnosis of schizophrenia (e.g., Vincent Van Gogh and John Nash), most patients do not show a higher level of creativity when compared to healthy people (Abraham et al., 2007). A recent meta-analysis from Acar et al. (2017) concludes that most evidence from empirical studies suggests that people with schizophrenia have, in fact, a worse creative performance, while very few studies have found a better creative performance in people with this disease (Glicksohn et al., 2001; Lauronen et al., 2004).

The study of the underlying neuroanatomical substrates of creativity in schizophrenia, nevertheless, is very scarce (Folley, 2006; Son et al., 2015), and it is only focused on specific brain areas and the fluency dimension instead of other aspects of creativity, such as originality. These studies (Folley, 2006; Son et al., 2015) did not find any significant results with creativity. In contrast, the amount of studies that have analyzed the underlying neuroanatomical substrates of creativity among healthy people has been growing during the last decade (Kenett et al., 2018). However, the brain correlates of creativity still remain inconclusive (Arden et al., 2010; Sawyer, 2011; Takeuchi and Kawashima, 2018). Some of the main brain regions that have been related to human creative thinking among healthy people include prefrontal, parietal, temporal, and subcortical areas (Arden et al., 2010; Boccia et al., 2015; Wu et al., 2015; Abraham et al., 2018; Japardi et al., 2018; Shi et al., 2018; Vartanian et al., 2018; Sun et al., 2019). Most of these findings have come from functional magnetic resonance imaging (fMRI) studies in healthy people.

Regarding structural brain correlates of creativity in healthy people, contradictory and inconclusive findings have been found (Takeuchi and Kawashima, 2018). Specifically, very few studies have analyzed the association between WM structural connectivity and creativity. Studies examining FA have found both positive (Takeuchi et al., 2010b) and negative associations (Jung et al., 2010), as well as non-significant associations (Takeuchi et al., 2016). For instance, Jung et al. (2010) found a negative correlation between a composite creativity score (including verbal and figural creativity tasks) and FA mainly within the left inferior frontal WM. In contrast, Takeuchi et al. (2010b) found positive correlations between total creativity (obtained from three verbal creativity tasks) and FA from the bilateral prefrontal cortex, corpus callosum, cingulate cortex, bilateral basal ganglia, bilateral temporoparietal junction, and the right inferior parietal lobe. Findings from Takeuchi et al. (2010b) suggest that multiple brain regions are involved in general creative thinking. Moreover, these results support the idea that both intra- and interhemispheric connections (especially the corpus callosum) as well as the frontal lobe underlie creative thinking. The role of intra- and interhemispheric connectivity is supported by the long-standing idea that integration of information and specifically integration of distant ideas are important for creativity (Katz, 1986; Hoppe and Kyle, 1990; Takeuchi et al., 2010b; Razumnikova and Volf, 2015). Concerning the frontal lobe, it has been proposed that WM integrity in this region facilitates multiple high-level cognitive functions, such as working memory and executive functions, which seem to underlie creative thinking (Takeuchi et al., 2010b). Additional evidence of the role of frontal lobe comes from studies that investigated the effect of transcranial direct current stimulation on creativity (Lucchiari et al., 2018).

Interestingly, brain regions identified by studies analyzing structural brain correlates of creativity in healthy individuals (Jung et al., 2010; Takeuchi et al., 2010b) are regions that have shown to be impaired in schizophrenia (Ellison-Wright and Bullmore, 2009; Bora et al., 2011; Stämpfli et al., 2019). This could suggest that creativity alterations found in this pathology could be at least partially due to WM abnormalities. In fact, structural connectivity alterations are a core characteristic of this disease (Stämpfli et al., 2019). Thus, WM alterations in schizophrenia are mainly circumscribed to frontal and temporal regions including mainly interhemispheric (corpus callosum), intrahemispheric (e.g., thalamic radiation, superior longitudinal fasciculus, inferior longitudinal fasciculus, inferior fronto-occipital fasciculus, and fornix), and projective fibers (corticospinal tract) (Ellison-Wright and Bullmore, 2009; Bora et al., 2011; Wheeler and Voineskos, 2014; Stämpfli et al., 2019).

Taking into account the structural connectivity alterations as well as the creativity impairment shown in schizophrenia, it seems relevant to study the WM correlates of creativity in this disease. As far as the authors are aware, to date, none of the previous studies have investigated whole brain WM correlates with different dimensions of creativity (such as fluency and originality) in schizophrenia. Therefore, the objective of this exploratory study was to assess whole brain WM correlates of several creativity dimensions in people with schizophrenia. Based on previous results from Takeuchi et al. (2010b), our hypothesis was that creativity would be positively associated mainly with the frontal lobe as well as with interhemispheric WM fibers.

\section{MATERIALS AND METHODS}

\section{Participants}

The sample consisted of 55 patients [ 47 men and eight women, mean age $41.22(S D=10.41)]$ diagnosed with schizophrenia and recruited from the Psychiatric Hospital of Álava and the Community Mental Health Services in Álava (Basque Country, Spain). All patients met the diagnostic criteria for schizophrenia according to the Structured Clinical Interview for Diagnostic and Statistical Manual of Mental Disorders, Fourth Edition, Text Revision (DSM-IV-TR; American Psychiatric Association, 2000).

Exclusion criteria consisted of: (a) clinical instability (total score in PANSS-Positive >19), (b) cognitive impairment secondary to another disease, (c) main diagnosis of a substance use disorder or presenting active drug consumption at the time of the study, (d) relevant modifications to the antipsychotic drug treatment in the previous 3 months, (e) diagnosis of an active major affective disorder, and (f) incompatibilities with magnetic resonance imaging: claustrophobia, metal implants in the body, or patients who were undergoing deep brain stimulation. The study protocol had the approval of the Clinical Research Ethics Committee of the Autonomous Region of the Basque Country (CEIC-E) in Spain (PI2017044). The trial was registered in clinicaltrials.gov (NCT03509597). All participants 
took part in the study voluntarily and provided their written informed consent. Participants did not receive any monetary reward for taking part in the study.

\section{Measures \\ Creativity}

Creativity was measured by means of two subtests from the Torrance Test of Creative Thinking (Torrance, 1966). From the Verbal Form of the test, the Unusual Uses subtest was administered. In this test, participants were asked to write all the unusual uses for cardboard boxes that they could think of. Three dimensions were measured: (1) originality, (2) fluency, and (3) flexibility. The Picture Completion subtest was used from the Figural Form of the test. In this activity, participants were asked to complete 10 unfinished figures, generating as many ideas as possible. Six dimensions were measured: (1) originality, (2) elaboration, (3) fluency, (4) resistance to premature closure, (5) abstractness of titles, and (6) figural creative strengths. Additionally, the flexibility dimension was measured using the criteria from the Spanish adaptation of the Torrance Test of Creative Thinking (Jiménez et al., 2007). Participants were given 4 min to complete each creative task. An expert neuropsychologist corrected all the tests.

\section{Clinical Symptoms}

Psychopathology was assessed with the PANSS (Kay et al., 1987). Positive Scale, Negative Scale, and General Psychopathology Scales were provided.

\section{Premorbid IQ}

Premorbid IQ was calculated with the Accentuation Reading Test (TAP) (Del Ser et al., 1997), a Spanish version of the National Adult Reading Test (Nelson and Willison, 1991). For the estimation of premorbid IQ, raw scores were converted using the full scale IQ of Gomar et al. (2011).

\section{Handedness}

Handedness was measured using the Edinburgh Handedness Inventory (Oldfield, 1971). In this self-reported questionnaire, participants were asked to indicate their preference of hand use for 10 everyday activities. Responses for each activity could be no preference ( 0 points), preference ( 1 point), and very strong preference ( 2 points). Handedness consistency was calculated using a formula (right - left/right + left), and the obtained scores ranged from 100 (perfectly right-handed) to -100 (perfectly left-handed). Mixed-handed were considered those participants who obtained scores ranging from -79 to 79 , and consistenthanded were those with scores ranging from -100 to -80 or from 80 to 100 .

\section{Image Acquisition and Analysis}

Diffusion-weighted images (DWIs) were obtained on a Philips 3T Achieva Dstream, in an axial orientation in an anterior-posterior phase direction using a singleshot $\mathrm{EPI}$ sequence $(\mathrm{TR}=7,540 \mathrm{~ms}$ and $\mathrm{TE}=76 \mathrm{~ms}$, matrix size $=120 \mathrm{~mm} \times 117 \mathrm{~mm} ;$ flip angle $=90^{\circ}$,
FOV $=240 \times 240 \times 130$, slice thickness $=2 \mathrm{~mm}$, no gap, 65 slices, acquisition time $=9^{\prime} 31^{\prime \prime}$, voxel size $=1.67 \times 1.67 \times 2.0$ ) with diffusion weighting in 32 uniformly distributed directions $\left(\mathrm{b}=1,000 \mathrm{~s} / \mathrm{mm}^{2}\right)$ and $1 \mathrm{~b}=0 \mathrm{~s} / \mathrm{mm}^{2}$.

The FMRIB Software Library (FSL) (Smith et al., 2004) version 5.0.11 $1^{1}$ was used for the preprocessing and analysis of diffusion data. First, each participant's images were concatenated and radiologically oriented. Next, data were corrected for head motion and eddy currents, brain extraction was performed using BET (Brain Extraction Tool) (Smith, 2002), and the diffusion gradients (bvecs) were rotated to be corrected accordingly. Then, FA, mean diffusivity (MD), radial diffusivity (RD), axial diffusivity (AD), and mode of anisotropy (MO) images were obtained by fitting a tensor model to the raw diffusion data using FDT (DTIFIT). Afterward, voxelwise statistical analysis of the data was carried out using Tract-Based Spatial Statistics (TBSS; Smith et al., 2006). FNIRT tool (Andersson et al., 2007a,b) was used to align all subjects' FA data into a common space by combining the non-linear transform to the target FA image with the affine transform from that target to MNI152 space. Later, the mean FA image was created using a threshold of 0.2 and thinned to create a mean FA skeleton, which represented the centers of all tracts common to the group. Each participant's aligned FA data were then projected onto this skeleton and the resulting data were fed into voxelwise cross-subject statistics. The "tbss non FA" script from TBSS was used to analyze $\mathrm{MD}, \mathrm{RD}, \mathrm{MO}$, and, $\mathrm{AD}$ data. This applies the original nonlineal registration to the $\mathrm{MD}, \mathrm{RD}, \mathrm{MO}$, or $\mathrm{AD}$ data, merges all subjects warped $\mathrm{MD}, \mathrm{RD}, \mathrm{MO}$, or $\mathrm{AD}$ data into a $4 \mathrm{D}$ file, then projects this onto the original mean FA skeleton, and creates the $4 \mathrm{D}$ projected data.

\section{Data Analyses}

All variables were tested for normality. Means and standard deviations were obtained using IBM SPSS version 26.0 (SPSS Inc., Chicago, IL, United States). As creativity variables did not follow a normal distribution, these were log-transformed (LN) and all variables were then transformed into $\mathrm{Z}$ scores. To examine the relationship between WM indexes and creativity, permutation-based inferences (5,000 permutations) with the threshold-free cluster enhancement (TFCE) correction method for multiple comparisons, including the - - T2 option, were carried out using FSL's Randomise Tool (Winkler et al., 2014). Based on findings from previous literature (Bartzokis et al., 2003; Schmithorst et al., 2005; Szeszko et al., 2008; White et al., 2011; Ryman et al., 2014; Samartzis et al., 2014; Kubota et al., 2015; Takeuchi et al., 2016; Jensen et al., 2019), sex, age, premorbid IQ, and medication were entered as covariates in the regression analysis. The statistical threshold was set at $p<0.05$ corrected for family wise error (FWE), with an extent threshold of 100 voxels. Effect sizes for each cluster of the correlations were calculated according to Cohen's $d$ formula. Cohen's $d$ of $0.20,0.50$, and 0.80 were considered small, medium, and large, respectively (Cohen, 1988). The maximum coordinates encompassed in the clusters and

\footnotetext{
${ }^{1}$ https://fsl.fmrib.ox.ac.uk/fsl/fslwiki/FSL
} 
additional significant regions were visually inspected and located and later labeled anatomically with the MRI Atlas of Human White Matter (Oishi et al., 2010) as well as the JHU-ICBMDTI-81 WM Labels and JHU White-Matter Tractography Atlas implemented in FSL.

Participants from the present study were assured that raw data would remain confidential and would not be shared. Therefore, data of the study are not available in the public domain.

\section{RESULTS}

\section{Sociodemographic, Clinical, and Creativity Data}

Sociodemographic and clinical characteristics of the sample as well as performance in verbal and figural creativity are shown in Table 1. Medication was transformed to chlorpromazine through the defined daily dose method (Leucht et al., 2016; Rothe et al., 2018). Standardized mean creativity scores of patients with schizophrenia based on a sample of healthy adults (obtained from Sampedro et al., 2019) were the following: $-0.55(S D=0.78)$ for verbal originality, $-0.78(S D=0.61)$ for verbal fluency, $-0.93(S D=0.69)$ for verbal flexibility, $-0.19(S D=0.64)$ for figural originality, $-0.20(S D=1.05)$ for figural fluency, -0.55 $(S D=0.81)$ for figural elaboration, $-0.27(S D=0.99)$ for figural flexibility, $-0.41(S D=1.02)$ for figural resistance to closure, $-0.78(S D=0.74)$ for figural abstractness of titles, and -0.84 $(S D=0.57)$ for figural creative strengths.

\section{Correlations Between Creativity and White Matter}

Significant positive correlations were found between mean WM FA and figural originality in two clusters (Table 2 and Figure 1). Significant negative correlations were not found between creativity dimensions and any brain region. In addition, there were no significant correlations between mean FA and verbal creativity dimensions.

The subdomain of figural creativity that correlated positively with mean WM FA was originality. More specifically, WM fibers corresponding to the peak level of significance were located in the right corticospinal tract (cerebral peduncle part) for cluster 1 and the right body of the corpus callosum for cluster 2 (Table 2). Other WM areas comprised in those same significant clusters included bilateral frontal (uncinate fasciculus, inferior fronto-occipital fasciculus, and WM adjacent to the superior frontal gyrus and orbitofrontal cortex), right temporal (uncinate fasciculus and WM adjacent to the inferior temporal gyrus), bilateral subcortical (fornix, anterior thalamic radiation, lenticular fasciculus, internal capsule, and external capsule), left brain stem (corticospinal tract), and bilateral interhemispheric (body, genu, and splenium of the corpus callosum) regions (FWE-corrected, $p<0.05$ ) (Figure 1). Effect sizes for these correlations were large for both clusters (Cohen's $d=1.13$ and Cohen's $d=0.92$ ). No significant correlations were found for $\mathrm{MD}, \mathrm{AD}, \mathrm{RD}$, or $\mathrm{MO}$ in this variable.

\section{DISCUSSION}

The main objective of this study was to assess whole brain WM correlates of different creativity dimensions in people with schizophrenia. Our findings showed that creative performance was positively related to WM mean FA adjacent to multiple brain regions, including frontal, temporal, subcortical, and brain stem areas as well as to interhemispheric WM fibers. Significant positive correlations were specially found in figural originality but not in any verbal creativity dimension. As a whole, these results are quite consistent with findings from Takeuchi et al. (2010b), in which total creativity correlated positively with WM adjacent to prefrontal areas, temporal and parietal lobes, basal ganglia, and corpus callosum in healthy people.

As expected, significant positive correlations were found between the figural originality subdomain of creativity and FA in the corpus callosum. This is congruent with findings from previous studies analyzing WM correlates of creativity (Takeuchi et al., 2010b; Zeng et al., 2017). WM integrity in the corpus callosum is thought to promote interhemispheric information processing and communication (Schulte et al., 2005). In addition, according to Heilman et al. (2003), different forms of knowledge and cognitive strategies can be combined through interhemispheric communication and, consequently, facilitate creative thinking. Therefore, these results support the long-held idea that interhemispheric communication is relevant for creative thinking (Katz, 1986; Bogen and Bogen, 1988; Hoppe and Kyle, 1990; Lindell, 2011).

Consistent with our hypothesis, results also showed significant positive correlations between the figural originality subdomain of creativity and WM tracts traversing the frontal lobe, such as the uncinate fasciculus and WM adjacent to the prefrontal cortex. These findings were also in line with both structural WM studies (Takeuchi et al., 2010b) and fMRI studies (Beaty et al., 2014, 2018; Japardi et al., 2018; Marron et al., 2018; Sun et al., 2019). Studies analyzing the effect of transcranial direct current stimulation on creativity also support the frontal lobe's role in creative performance (Lucchiari et al., 2018). Moreover, creativity has been particularly related to the prefrontal cortex (Benedek et al., 2014a; Beaty et al., 2018; Lucchiari et al., 2018). It has been suggested that several higher-order cognitive functions, such as working memory and executive functions, are required for creative thinking since they enable, for instance, the maintenance of novel information in an elevated state of activity (de Dreu et al., 2012) or the inhibition of salient but unoriginal ideas (Benedek et al., 2014b). WM integrity in the frontal lobe seems to be involved in these higher-order cognitive abilities (Charlton et al., 2010; Jacobs et al., 2013) and, therefore, in creative thinking (Takeuchi et al., 2010b). Figural originality was additionally related to WM adjacent to the orbitofrontal cortex, including the straight and orbital gyrus. The orbitofrontal cortex has been related to decision-making (Padoa-Schioppa and Conen, 2017), to attentional control (Ohtani et al., 2017), and to creativity in men (Abraham et al., 2014). According to Schuck et al. (2016), the orbitofrontal cortex is a region involved in the flexible up-todate representation of information that is relevant to a given task, thus allowing decision-making. The association of this region 
TABLE 1 | Sociodemographic, clinical, and creativity data of the sample.

Patients with schizophrenia $(N=55) M(S D)$

Sociodemographic and clinical characteristics
Sex (male)
Age (years)
Education years
Handedness
Right-handedness
Mixed
Age of onset
Previous hospitalizations
Premorbid IQ
Medication dosage (chlorpromazine equivalent doses, mg/day)
PANSS Positive
PANSS Negative
PANSS General
PANSS Total
Creativity assessment
Verbal originality
Verbal fluency
Verbal flexibility
Figural originality
Figural fluency
Figural elaboration
Figural flexibility
Figural resistance to Closure
Figural abstractness of Titles
Figural creative strengths

M, mean; SD, standard deviation; PANSS, Positive and Negative Syndrome Scale.

TABLE 2 | Correlation analysis between figural originality and mean fractional anisotropy (FA) showing two significant clusters.

\begin{tabular}{|c|c|c|c|c|c|c|c|c|}
\hline & Brain regions & Cluster size (voxels) & \multicolumn{6}{|c|}{ FSL coordinates } \\
\hline Cluster 1 & Corticospinal tract (cerebral peduncle part) (R) & 7,551 & 77 & 112 & 56 & 3.91 & 0.024 & 1.13 \\
\hline Cluster 2 & Body of the corpus callosum (R) & 5,681 & 83 & 100 & 99 & 3.25 & 0.025 & 0.92 \\
\hline
\end{tabular}

Cluster size denotes the extent of the cluster of significant voxels. FMRIB Software Library (FSL) coordinates indicate: $x$ increases from left to right; $y$ increases from posterior to anterior; and $z$ increases from inferior to superior. Regions represent the maximum significant correlation coordinate encompassed in the given cluster. $R$, right.

with originality makes sense, since representing all the relevant information for a creative task seems to be important in order to find or decide which is the most original idea. Furthermore, this region seems to be particularly altered in patients with schizophrenia (Takayanagi et al., 2010; Kanahara et al., 2013).

In addition to WM from the corpus callosum and frontal lobe, we also found a relationship between the figural originality subdomain of creativity and intrahemispheric WM fibers located in several association regions from the temporal lobe, specifically, the uncinate fasciculus and WM adjacent to the inferior temporal gyrus. This indicates that intrahemispheric communication may also be required for creative thinking through the integration of remote information belonging to multiple regions that are involved in different domains (Takeuchi et al., 2010b). Besides, the involvement of the temporal lobe in creative thinking is consistent with other structural (Takeuchi et al., 2010b) and functional studies (Benedek et al., 2014a; Beaty et al., 2015) and could be due to its relation with memory retrieval. Memory retrieval is required for creative thinking, since original ideas seems to be generated through the direction of attention to internal knowledge representations, the controlled retrieval, and the recombination of this stored knowledge (Benedek et al., 2014a).

White matter tracts located in subcortical regions were also associated with creativity. They included the fornix, internal capsule, external capsule, anterior thalamic radiation, and lenticular fasciculus. These regions could be involved in creativity due to their implication in the dopaminergic system (Takeuchi et al., 2010a,b; Jauk et al., 2015). An increased WM integrity in these subcortical regions is thought to increase functional 


\section{A Cluster 1. Right hemisphere $x=13 y=-14 z=-16$}

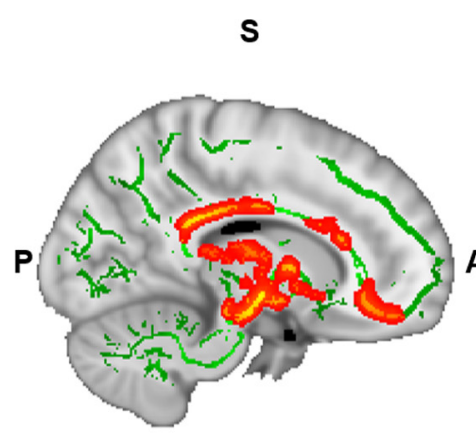

I

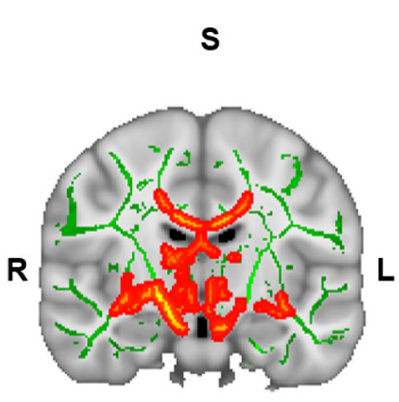

I

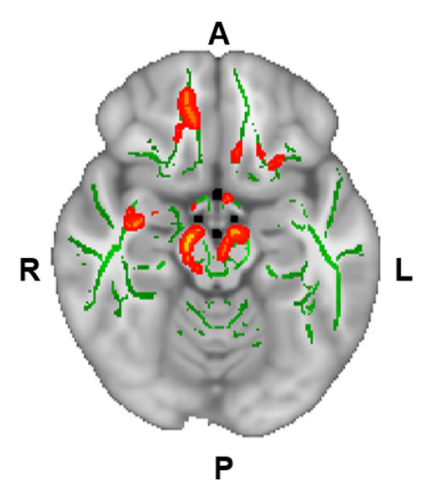

$\mathbf{P}$

B Cluster 2. Right hemisphere $x=7 y=-26 z=27$

s

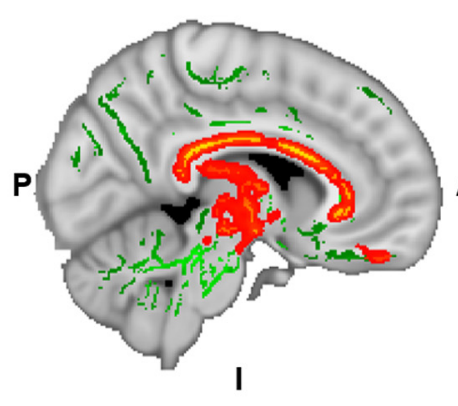

S

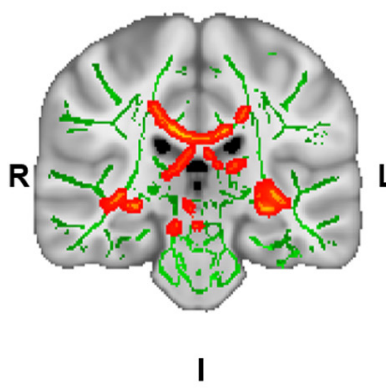

$\mathbf{L} \mathbf{R}$

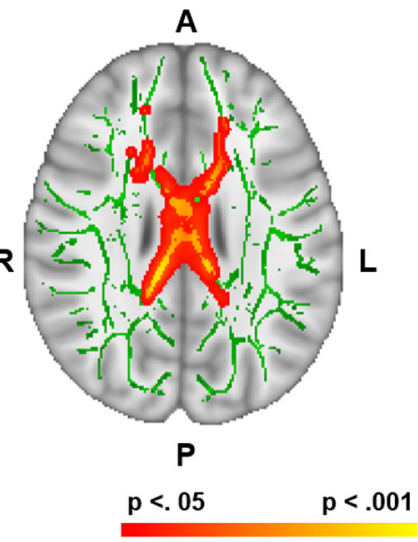

FIGURE 1 | Correlations between figural originality and white matter (WM) mean fractional anisotropy (FA) in the two significant clusters [(A) for cluster 1 and (B) for cluster 2]. Correlations are significant at $p<0.05$ corrected for family wise error (FWE). Significant WM regions are shown in red-yellow; the WM skeleton is shown in green. Significant voxels are thickened for easier visualization. S, superior; I, inferior; A, anterior; P, posterior; L, left; R, right. Coordinates are shown in Montreal Neurological Institute (MNI) space. The region located in the coordinates that showed the maximum significance was the right cerebral peduncle of the corticospinal tract for cluster 1 (A) and the right body of the corpus callosum for cluster 2 (B).

connectivity and, consequently, improve some higher-order frontal lobe abilities that underlie creative thinking, such as cognitive flexibility, through the regulation of the dopaminergic system (Takeuchi et al., 2010b).

Finally, a positive correlation was found between creativity and the corticospinal tract in its cerebral peduncle part. Although this region has not been related to creativity, the cerebral peduncle is a main component of the cortico-ponto-cerebellothalamo-cortical loop and it is believed to connect the cerebellum with the neocortex (Hüttlova et al., 2014). The cerebral peduncle and the corticospinal tract are regions involved not only in movement but also in cognitive processes such as coordination and information processing (Picard et al., 2008). Additional evidence comes from several studies that have shown that the cerebellum is highly involved in creativity through cerebralcerebellar interactions that underlie implicit manipulation of mental representation, which in turn promote the development of more creative ideas (Saggar et al., 2015, 2017). Moreover, cerebral peduncles and the corticospinal tract are particularly impaired in schizophrenia (Hüttlova et al., 2014; Stämpfli et al., 2019). Therefore, decreased WM integrity in these areas could possibly promote an impairment in some cognitive functions such as creativity.

Although we found some greater correlations in regions from the right hemisphere, in general, there were bilateral correlations with creativity. In fact, in spite of the long-held belief that the right hemisphere is mainly responsible for creative thinking (Harnad, 1972), nowadays, results from most brain imaging studies suggest that creativity requires both hemispheres (Beaty et al., 2014, 2016; Wu et al., 2015). Moreover, it seems that it is not so much a matter of the left or right hemisphere, but rather a complex set of intra- and interhemispheric connections within the whole brain (Corballis, 2018).

It is worth mentioning that we did not find significant correlations with all dimensions and, specifically, with verbal creativity. The absence of significant correlations in various 
variables could be partially due to the sample size. In any case, several of the few studies that analyze WM FA correlates of creativity have not found significant correlations either in patients with schizophrenia (Folley, 2006; Son et al., 2015) or in healthy people (Takeuchi et al., 2016). Other studies analyzing WM integrity (Zeng et al., 2017) or gray matter volume (Jauk et al., 2015) also found significant correlations with originality but not with fluency. An additional possible explanation for these results could be the language impairment present in schizophrenia (Mitchell and Crow, 2005; Docherty et al., 2011), which makes verbal creativity tasks particularly challenging for these patients. In consequence, the general performance in verbal dimensions may have been low, with a lower variability of the data among the sample than in figural creativity, making it more difficult to correlate with WM integrity. More research is required to analyze brain correlates of verbal creativity in schizophrenia, controlling for the possible influence of other cognitive variables, such as verbal memory.

Taken together, this study provides initial data for brain WM correlates of creativity in schizophrenia. Overall, results suggest that both intrahemispheric communication and interhemispheric communication seem to be required for creative thinking, allowing the integration of remote associations of ideas. Our findings support the role of the frontal lobe in creative thinking but also indicate the relevance of other brain regions such as the corticospinal tract. Results from this study are similar to those from Takeuchi et al. (2010b) carried out with healthy people, which suggests that similar brain regions are involved in creative thinking of people with schizophrenia and healthy people. This idea is in line with the creative cognition approach, which considers that creativity emerges from the application of basic cognitive functions to already existing knowledge structures and therefore assumes that creative capacity is a normative human characteristic (Ward, 2007). Given that WM alteration is a core characteristic of schizophrenia (Stämpfli et al., 2019) and that many of the brain regions that correlated with creativity in our study are brain regions that are particularly impaired in this disease (e.g., the corpus callosum, fornix, or corticospinal tract), we could hypothesize that the impairment in creative performance of people with schizophrenia is related to an alteration in WM integrity. Nevertheless, this idea must be considered with caution, since we did not compare WM integrity of our sample with healthy controls.

\section{Limitations}

The present study has several limitations that should be considered. First, we did not include healthy controls to check whether there was an impairment in WM integrity. However, our interpretations of the results were based on previous evidence, which has shown that people with schizophrenia have WM alterations in specific brain regions (Ellison-Wright and Bullmore, 2009; Bora et al., 2011; Stämpfli et al., 2019). Future studies should explore WM correlates of creativity in schizophrenia compared to healthy controls to analyze whether there are differences in the brain areas related to creativity. Second, the small sample size limits generalization of results, so more studies should be conducted with larger samples and results of this study should be considered with caution. Finally, other variables such as clinical symptoms and cognitive capacity in several domains like executive functions, attention, or working memory could have an effect on creative performance; hence, future studies should control the influence of these variables.

\section{Conclusion}

As far as the authors are aware, this is the first study analyzing whole brain WM correlates of different creativity dimensions in schizophrenia. We consider that this study provides relevant preliminary data that could, on the one hand, shed light on the long-standing and controversial association between psychosis and creativity and, on the other hand, contribute to fill a gap in research on the neuroscience of human creativity. Results suggest that multiple brain regions are involved in creative thinking, which makes sense due to the complexity and multifaceted nature of this higher-order cognitive function. Yet more research is needed in order to understand the neural bases of creativity in both schizophrenia and healthy people. Understanding the brain correlates of creativity in schizophrenia has relevant implications for the treatment of mental disorders and for the enhancement of this ability that is essential for the survival and enrichment of humankind (Carson, 2014).

\section{DATA AVAILABILITY STATEMENT}

The datasets for this article are not publicly available because participants from the present study were assured raw data would remain confidential and would not be shared.

\section{ETHICS STATEMENT}

The studies involving human participants were reviewed and approved by Clinical Research Ethics Committee of the Autonomous Region of the Basque Country (CEIC-E) in Spain (PI2017044). The patients/participants provided their written informed consent to participate in this study.

\section{AUTHOR CONTRIBUTIONS}

NO, NI-B, JP, and PS designed the study and wrote the protocol. AS, AC-Z, PS, NI-Y, and CP performed the clinical, neuropsychological, and neuroimaging assessments. AS and JP managed the literature searches. AS, JP, and AG-G undertook the statistical analysis. AS and JP wrote the first draft of the manuscript. All authors contributed to the writing and revision of the manuscript and approved the final manuscript.

\section{FUNDING}

This study has been supported by the Spanish Ministry of Economy and Competitiveness (PI16/01022) and the Department of Education and Science of the Basque Government (Team A) (IT946-16). AS was supported by a fellowship from the Fundación Tatiana Pérez de Guzmán 
el Bueno. AG-G was supported by a fellowship from the Education, Language, Politics and Culture Department of the Basque Government (PRE_2015_1_0444). The funding agencies had no role in the study design, data collection and analysis, decision to publish, or preparation of the manuscript.

\section{REFERENCES}

Abraham, A., Rutter, B., Bantin, T., and Hermann, C. (2018). Creative conceptual expansion: a combined fMRI replication and extension study to examine individual differences in creativity. Neuropsychologia 118, 29-39. doi: 10.1016/ j.neuropsychologia.2018.05.004

Abraham, A., Thybusch, K., Pieritz, K., and Hermann, C. (2014). Gender differences in creative thinking: behavioral and fMRI findings. Brain Imaging Behav. 8, 39-51. doi: 10.1007/s11682-013-9241-4

Abraham, A., Windmann, S., McKenna, P., and Güntürkün, O. (2007). Creative thinking in schizophrenia: the role of executive dysfunction and symptom severity. Cogn. Neuropsychiatry 12, 235-258. doi: 10.1080/13546800601046714

Acar, S., Chen, X., and Cayirdag, N. (2017). Schizophrenia and creativity: a metaanalytic review. Schizophr. Res. 195, 23-31. doi: 10.1016/j.schres.2017.08.036

American Psychiatric Association (2000). Diagnostic and Statistical Manual of Mental Disorders, 4th Edn. Washington, DC: American Psychiatric Association.

Andersson, J., Jenkinson, M., and Smith, S. (2007a). Non-linear Optimisation. FMRIB Technical Report TR07JA1. Oxford: University of Oxford FMRIB Centre.

Andersson, J., Jenkinson, M., and Smith, S. M. (2007b). Non-linear Registration, aka Spatial Normalisation. FMRIB Technical Report TR07JA2. Oxford: FMRIB Centre.

Arden, R., Chavez, R. S., Grazioplene, R., and Jung, R. E. (2010). Neuroimaging creativity: a psychometric view. Behav. Brain Res. 214, 143-156. doi: 10.1016/j. bbr.2010.05.015

Bartzokis, G., Cummings, J. L., Sultzer, D., Henderson, V. W., Nuechterlein, K., and Mintz, J. (2003). White matter structural integrity in healthy aging adults and patients with Alzheimer disease: a magnetic resonance imaging study. Arch. Neurol. 60, 393-398.

Beaty, R. E., Benedek, M., Kaufman, S. B., and Silvia, P. J. (2015). Default and executive network coupling supports creative idea production. Sci. Rep. 5:10964. doi: 10.1038/srep10964

Beaty, R. E., Benedek, M., Silvia, P. J., and Schacter, D. L. (2016). Creative cognition and brain network dynamics. Trends Cogn. Sci. 20, 87-95. doi: 10.1016/j.tics. 2015.10.004

Beaty, R. E., Benedek, M., Wilkins, R. W., Jauk, E., Fink, A., Silvia, P. J., et al. (2014). Creativity and the default network: a functional connectivity analysis of the creative brain at rest. Neuropsychologia 64, 92-98. doi: 10.1016/j. neuropsychologia.2014.09.019

Beaty, R. E., Kenett, Y. N., Christensen, A. P., Rosenberg, M. D., Benedek, M., Chen, Q., et al. (2018). Robust prediction of individual creative ability from brain functional connectivity. Proc. Natl. Acad. Sci. U.S.A. 115, 1087-1092. doi: 10.1073/pnas.1713532115

Benedek, M., Jauk, E., Fink, A., Koschutnig, K., Reishofer, G., Ebner, F., et al. (2014a). To create or to recall? Neural mechanisms underlying the generation of creative new ideas. Neuroimage 88, 125-133. doi: 10.1016/j.neuroimage.2013. 11.021

Benedek, M., Jauk, E., Sommer, M., Arendasy, M., and Neubauer, A. C. (2014b). Intelligence, creativity, and cognitive control: the common and differential involvement of executive functions in intelligence and creativity. Intelligence 46, 73-83. doi: 10.1016/j.intell.2014.05.007

Boccia, M., Piccardi, L., Palermo, L., Nori, R., and Palmiero, M. (2015). Where do bright ideas occur in ourbrain? Meta-analytic evidence from neuroimaging studies of domain-specific creativity. Front. Psychol. 6:1195. doi: 10.3389/fpsyg. 2015.01195

Bogen, J. E., and Bogen, G. M. (1988). Creativity and the corpus callosum. Psychiatr. Clin. North Am. 11, 293-301. doi: 10.1016/S0193-953X(18)30481-7

Bora, E., Fornito, A., Radua, J., Walterfang, M., Seal, M., Wood, S. J., et al. (2011). Neuroanatomical abnormalities in schizophrenia: a multimodal voxelwise

\section{ACKNOWLEDGMENTS}

The authors thank all the participants and clinical clusters who were involved in the study as well as the English language editing service.

meta-analysis and meta-regression analysis. Schizophr. Res. 127, 46-57. doi: 10.1016/j.schres.2010.12.020

Carson, S. H. (2014). "The shared vulnerability model of creativity and psychopathology," in Creativity and Mental Illness, ed. J. C. Kaufman (New York, NY: Cambridge University Press), 253-280. doi: 10.1017/ CBO9781139128902

Charlton, R. A., Barrick, T. R., Lawes, I. N. C., Markus, H. S., and Morris, R. G. (2010). White matter pathways associated with working memory in normal aging. Cortex 46, 474-489. doi: 10.1016/j.cortex.2009.07.005

Cohen, J. (1988). Statistical Power Analysis for the Behavioral Sciences. Abingdon: Routledge.

Corballis, M. C. (2018). "Laterality and creativity: a false trail?," in The Cambridge Handbook of the Neuroscience of Creativity, eds R. E. Jung and O. Vartanian (Cambridge: Cambridge University Press), 50-57. doi: 10.1017/9781316556 238.004

de Dreu, C. K. W., Nijstad, B. A., Baas, M., Wolsink, I., and Roskes, M. (2012). Working memory benefits creative insight, musical improvisation, and original ideation through maintained task-focused attention. Personal. Soc. Psychol. Bull. 38, 656-669. doi: 10.1177/0146167211435795

Del Ser, T., Gonzalez-Montalvo, J. I., Martinez-Espinosa, S., Delgado-Villapalos, C., and Bermejo, F. (1997). Estimation of premorbid intelligence in Spanish people with the word accentuation test and its application to the diagnosis of dementia. Brain Cogn. 33, 343-356. doi: 10.1006/brcg.1997.0877

Docherty, A. R., Berenbaum, H., and Kerns, J. G. (2011). Alogia and formal thought disorder: differential patterns of verbal fluency task performance. J. Psychiatr. Res. 45, 1352-1357. doi: 10.1016/j.jpsychires.2011.04.004

Ellison-Wright, I., and Bullmore, E. (2009). Meta-analysis of diffusion tensor imaging studies in schizophrenia. Schizophr. Res. 108, 3-10. doi: 10.1016/j. schres.2008.11.021

Folley, B. S. (2006). The Cognitive Neuroscience of Creative Thinking in the Schizophrenia. Ph.D. thesis, Vanderbilt University Libraries, Nashville, TN.

Glicksohn, J., Alon, A., Perlmutter, A., and Purisman, R. (2001). Symbolic and syncretic cognition among schizophrenics and visual artists. Creat. Res. J. 13, 185-195. doi: 10.1207/S15326934CRJ1302

Gomar, J. J., Ortiz-Gil, J., McKenna, P. J., Salvador, R., Sans-Sansa, B., Sarró, S., et al. (2011). Validation of the word accentuation test (TAP) as a means of estimating premorbid IQ in Spanish speakers. Schizophr. Res. 128, 175-176. doi: 10.1016/j.schres.2010.11.016

Harnad, S. R. (1972). Creativity, lateral saccades and the nondominant hemisphere. Percept. Mot. Skills 34, 653-654. doi: 10.2466/pms.1972.34.2.653

Heilman, K. M., Nadeau, S. E., and Beversdorf, D. O. (2003). Neurocase: the neural basis of cognition creative innovation: possible brain mechanisms creative innovation: possible brain mechanisms. Neurocase 9, 369-379. doi: 10.1076/ neur.9.5.369.16553

Hoppe, K. D., and Kyle, N. L. (1990). Dual brain, creativity, and health. Creat. Res. J. 3, 150-157. doi: 10.1080/10400419009534348

Hüttlova, J., Kikinis, Z., Kerkovsky, M., Bouix, S., Vu, M. A., Makris, N., et al. (2014). Abnormalities in myelination of the superior cerebellar peduncle in patients with schizophrenia and deficits in movement sequencing. Cerebellum 13, 415-424. doi: 10.1007/s12311-014-0550-y

Jacobs, H. I. L., Leritz, E. C., Williams, V. J., Van Boxtel, M. P. J., Elst, W., Van Der Jolles, J., et al. (2013). Association between white matter microstructure, executive functions, and processing speed in older adults: the impact of vascular health. Hum. Brain Mapp. 34, 77-95. doi: 10.1002/hbm. 21412

Japardi, K., Bookheimer, S., Knudsen, K., Ghahremani, D. G., and Bilder, R. M. (2018). Functional magnetic resonance imaging of divergent and convergent thinking in Big-C creativity. Neuropsychologia 118, 59-67. doi: 10.1016/j. neuropsychologia.2018.02.017 
Jauk, E., Neubauer, A. C., Dunst, B., Fink, A., and Benedek, M. (2015). Gray matter correlates of creative potential: a latent variable voxel-based morphometry study. Neuroimage 111, 312-320. doi: 10.1016/j.neuroimage.2015.02.002

Jensen, M. H., Bak, N., Rostrup, E., Nielsen, M., Pantelis, C., Glenthøj, B. Y., et al. (2019). The impact of schizophrenia and intelligence on the relationship between age and brain volume. Schizophr. Res. Cogn. 15, 1-6. doi: 10.1016/j. scog.2018.09.002

Jiménez, J. E., Artiles, C., Rodríguez, M., and García, E. (2007). Adaptación y Baremación del test de Pensamiento Creativo de Torrance: Expresión Figurada. Canarias: Consejería.

Jung, R. E., Grazioplene, R., Caprihan, A., Chavez, R. S., and Haier, R. J. (2010). White matter integrity, creativity, and psychopathology: disentangling constructs with diffusion tensor imaging. PLoS One 5:e9818. doi: 10.1371/ journal.pone.0009818

Kanahara, N., Sekine, Y., Haraguchi, T., Uchida, Y., Hashimoto, K., Shimizu, E., et al. (2013). Orbitofrontal cortex abnormality and deficit schizophrenia. Schizophr. Res. 143, 246-252. doi: 10.1016/j.schres.2012.11.015

Katz, A. N. (1986). The Relationships between creativity and cerebral hemisphericity for creative architects, scientists, and mathematicians. Empir. Stud. Arts 4, 97-108. doi: 10.2190/6nhb-pev0-25kp-ukec

Kay, S. R., Fiszbein, A., and Opler, L. A. (1987). The positive and negative syndrome scale (PANSS) for schizophrenia. Schizophr. Bull. 13, 261-276. doi: 10.1093/ schbul/13.2.261

Kenett, Y. N., Medaglia, J. D., Beaty, R. E., Chen, Q., Betzel, R. F., ThompsonSchill, S. L., et al. (2018). Driving the brain towards creativity and intelligence: a network control theory analysis. Neuropsychologia 118, 79-90. doi: 10.1016/j. neuropsychologia.2018.01.001

Kubota, M., Van Haren, N. E. M., Haijma, S. V., Schnack, H. G., Cahn, W., Pol, H. E. H., et al. (2015). Association of IQ changes and progressive brain changes in patients with schizophrenia. JAMA Psychiatry 72, 803-812. doi: 10.1001/ jamapsychiatry.2015.0712

Lauronen, E., Veijola, J., Isohanni, I., Jones, P. B., Nieminen, P., and Isohanni, M. (2004). Links between creativity and mental disorder. Psychiatry Interpers. Biol. Process. 67, 81-98. doi: 10.1521/psyc.67.1.81.31245

Leucht, S., Samara, M., Heres, S., and Davis, J. M. (2016). Dose equivalents for antipsychotic drugs: the DDD method. Schizophr. Bull. 42, S90-S94. doi: 10. 1093/schbul/sbv167

Lindell, A. K. (2011). Lateral thinkers are not so laterally minded: hemispheric asymmetry, interaction, and creativity. Laterality 16, 479-498. doi: 10.1080/ 1357650X.2010.497813

Lucchiari, C., Sala, P. M., and Vanutelli, M. E. (2018). Promoting creativity through transcranial direct current stimulation (tDCS). A critical review. Front. Behav. Neurosci. 12:167. doi: 10.3389/fnbeh.2018.00167

Marron, T. R., Lerner, Y., Berant, E., Kinreich, S., Shapira-Lichter, I., Hendler, T., et al. (2018). Chain free association, creativity, and the default mode network. Neuropsychologia 118, 40-58. doi: 10.1016/j.neuropsychologia.2018.03.018

Mitchell, R. L. C., and Crow, T. J. (2005). Right hemisphere language functions and schizophrenia: the forgotten hemisphere? Brain 128, 963-978. doi: 10.1093/ brain/awh466

Nelson, H. E., and Willison, J. (1991). National Adult Reading Test (NART). Windsor: NFER-Nelson.

Ohtani, T., Nestor, P. G., Bouix, S., Newell, D., Melonakos, E. D., McCarley, R. W., et al. (2017). Exploring the neural substrates of attentional control and human intelligence: diffusion tensor imaging of prefrontal white matter tractography in healthy cognition. Neuroscience 341, 52-60. doi: 10.1016/j.neuroscience.2016. 11.002

Oishi, K., Faria, A., van Zijl, P., and Mori, S. (2010). MRI Atlas of Human White Matter. Cambridge, MA: Academic Press.

Oldfield, R. C. (1971). The assessment of handedness: the Edinburgh inventory. Neuropsychologia 9, 97-113. doi: 10.1016/0028-3932(71)90067-4

Padoa-Schioppa, C., and Conen, K. E. (2017). Orbitofrontal cortex: a neural circuit for economic decisions. Neuron 37, 784-790. doi: 10.1183/09031936.00063810

Picard, H., Amado, I., Mouchet-Mages, S., Olié, J. P., and Krebs, M. O. (2008). The role of the cerebellum in schizophrenia: an update of clinical, cognitive, and functional evidences. Schizophr. Bull. 34, 155-172. doi: 10.1093/schbul/sbm049

Plucker, J. A., Esping, A., Kaufman, J. C., and Avitia, M. J. (2015). "Creativity and intelligence," in Handbook of Intelligence: Evolutionary Theory, Historical Perspective, and Current Concepts, eds S. Goldstein, D. Princiotta, and J. A.
Naglieri (New York, NY: Springer Nature), 283-291. doi: 10.1007/978-1-49391562-0

Razumnikova, O. M., and Volf, N. V. (2015). Creativity-related hemispheric selective processing: correlations on global and local levels of attentional set. Creat. Res. J. 27, 394-399. doi: 10.1080/10400419.2015.1087272

Rindermann, H., and Neubauer, A. C. (2004). Processing speed, intelligence, creativity, and school performance: testing of causal hypotheses using structural equation models. Intelligence 32, 573-589. doi: 10.1016/j.intell.2004.06.005

Rothe, P. H., Heres, S., and Leucht, S. (2018). Dose equivalents for second generation long-acting injectable antipsychotics: the minimum effective dose method. Schizophr. Res. 193, 23-28. doi: 10.1016/j.schres.2017.07.033

Ryman, S. G., van den Heuvel, M. P., Yeo, R. A., Caprihan, A., Carrasco, J., Vakhtin, A. A., et al. (2014). Sex differences in the relationship between white matter connectivity and creativity. Neuroimage 101, 380-389. doi: 10.1016/j. neuroimage.2014.07.027

Saggar, M., Quintin, E. M., Bott, N. T., Kienitz, E., Chien, Y. H., Hong, D. W. C., et al. (2017). Changes in brain activation associated with spontaneous improvization and figural creativity after design-thinking-based training: a longitudinal fMRI study. Cereb. Cortex 27, 3542-3552. doi: 10.1093/cercor/ bhw171

Saggar, M., Quintin, E. M., Kienitz, E., Bott, N. T., Sun, Z., Hong, W. C., et al. (2015). Pictionary-based fMRI paradigm to study the neural correlates of spontaneous improvisation and figural creativity. Sci. Rep. 5:10894. doi: 10. 1038/srep 10894

Samartzis, L., Dima, D., Fusar-Poli, P., and Kyriakopoulos, M. (2014). White matter alterations in early stages of schizophrenia: a systematic review of diffusion tensor imaging studies. J. Neuroimaging 24, 101-110. doi: 10.1111/j.1552-6569. 2012.00779.x

Sampedro, A., Peña, J., Ibarretxe-bilbao, N., Sánchez, P., Iriarte-yoller, N., Ledesma-gonzález, S., et al. (2019). Mediating role of cognition and social cognition on creativity among patients with schizophrenia and healthy controls: revisiting the shared vulnerability model. Psychiatry Clin. Neurosci. 74, 1-7. doi: $10.1111 /$ pcn.12954/full

Sawyer, K. (2011). The cognitive neuroscience of creativity: a critical review. Creat. Res. J. 23, 137-154. doi: 10.1080/10400419.2011.571191

Schmithorst, V. J., Wilke, M., Dardzinski, B. J., and Holland, S. K. (2005). Cognitive functions correlate with white matter architecture in a normal pediatric population. Hum. Brain Mapp. 26, 139-147. doi: 10.1002/hbm.20149

Schuck, N. W., Cai, M. B., Wilson, R. C., and Niv, Y. (2016). Human orbitofrontal cortex represents a cognitive map of state space. Neuron 91, 1402-1412. doi: 10.1016/j.neuron.2016.08.019

Schulte, T., Sullivan, E. V., Müller-Oehring, E. M., Adalsteinsson, E., and Pfefferbaum, A. (2005). Corpus callosal microstructural integrity influences interhemispheric processing: a diffusion tensor imaging study. Cereb. Cortex 15, 1384-1392. doi: 10.1093/cercor/bhi020

Shi, L., Sun, J., Xia, Y., Ren, Z., Chen, Q., Wei, D., et al. (2018). Large-scale brain network connectivity underlying creativity in restingstate and task fMRI: cooperation between default network and frontalparietal network. Biol. Psychol. 135, 102-111. doi: 10.1016/j.biopsycho.2018. 03.005

Smith, S. M. (2002). Fast robust automated brain extraction. Hum. Brain Mapp. 17, 143-155. doi: 10.1002/hbm.10062

Smith, S. M., Jenkinson, M., Johansen-berg, H., Rueckert, D., Nichols, T. E., Mackay, C. E., et al. (2006). Tract-based spatial statistics: voxelwise analysis of multi-subject diffusion data. Neuroimage 31, 1487-1505. doi: 10.1016/j. neuroimage.2006.02.024

Smith, S. M., Jenkinson, M., Woolrich, M. W., Beckmann, C. F., Behrens, T. E. J., Johansen-berg, H., et al. (2004). Advances in functional and structural MR image analysis and implementation as FSL. Neuroimage 23, 208-219. doi: 10. 1016/j.neuroimage.2004.07.051

Son, S., Kubota, M., Miyata, J., Fukuyama, H., Aso, T., Urayama, I., et al. (2015). Creativity and positive symptoms in schizophrenia revisited: structural connectivity analysis with diffusion tensor imaging. Schizophr. Res. 164, 221226. doi: 10.1016/j.schres.2015.03.009

Stämpfli, P., Sommer, S., Manoliu, A., Burrer, A., Schmidt, A., Herdener, M., et al. (2019). Subtle white matter alterations in schizophrenia identified with a new measure of fiber density. Sci. Rep. 9:4636. doi: 10.1038/s41598-01940070-2 
Sun, J., Liu, Z., Rolls, E. T., Chen, Q., Yao, Y., Yang, W., et al. (2019). Verbal creativity correlates with the temporal variability of brain networks during the resting state. Cereb. Cortex 29, 1047-1058. doi: 10.1093/cercor/bhy010

Szeszko, P. R., Robinson, D. G., Ashtari, M., Vogel, J., Betensky, J., Sevy, S., et al. (2008). Clinical and neuropsychological correlates of white matter abnormalities in recent onset schizophrenia. Neuropsychopharmacology 33, 976-984. doi: 10.1038/sj.npp.1301480

Takayanagi, Y., Takahashi, T., Orikabe, L., Masuda, N., Mozue, Y., Nakamura, K., et al. (2010). Volume reduction and altered sulco-gyral pattern of the orbitofrontal cortex in first-episode schizophrenia. Schizophr. Res. 121, 55-65. doi: 10.1016/j.schres.2010.05.006

Takeuchi, H., and Kawashima, R. (2018). "Structural studies of creativity measured by divergent thinking," in The Cambridge Handbook of the Neuroscience of Creativity, eds R. E. Jung and O. Vartanian (Cambridge: Cambridge University Press), 451-463. doi: 10.1017/9781316556238.026

Takeuchi, H., Taki, Y., Nouchi, R., Yokoyama, R., Kotozaki, Y., Nakagawa, S., et al. (2016). Creative females have larger white matter structures: evidence from a large sample study. Hum. Brain Mapp. 38, 414-430. doi: 10.1002/hbm.23369

Takeuchi, H., Taki, Y., Sassa, Y., Hashizume, H., Sekiguchi, A., Fukushima, A., et al. (2010a). Regional gray matter volume of dopaminergic system associate with creativity: evidence from voxel-based morphometry. Neuroimage 51, 578-585. doi: 10.1016/j.neuroimage.2010.02.078

Takeuchi, H., Taki, Y., Sassa, Y., Hashizume, H., Sekiguchi, A., Fukushima, A., et al. (2010b). White matter structures associated with creativity: evidence from diffusion tensor imaging. Neuroimage 51, 11-18. doi: 10.1016/j.neuroimage. 2010.02.035

Thys, E., Sabbe, B., and De Hert, M. (2013). Creativity and psychiatric Illness: the search for a missing link - an historical context for current research. Psychopathology 46, 136-144. doi: 10.1159/000339458

Torrance, E. P. (1966). Torrance Tests of Creative Thinking. Bensenville, IL: Scholastic.

Vartanian, O., Beatty, E. L., Smith, I., Blackler, K., Lam, Q., and Forbes, S. (2018). One-way traffic: the inferior frontal gyrus controls brain activation in the middle temporal gyrus and inferior parietal lobule during divergent thinking. Neuropsychologia 118, 68-78. doi: 10.1016/j.neuropsychologia.2018.02.024

Ward, T. B. (2007). Creative cognition as a window on creativity. Methods 42, 28-37. doi: 10.1016/j.ymeth.2006.12.002

Wheeler, A. L., and Voineskos, A. N. (2014). A review of structural neuroimaging in schizophrenia: from connectivity to connectomics. Front. Hum. Neurosci. 8:653. doi: 10.3389/fnhum.2014.00653

White, T., Magnotta, V. A., Bockholt, H. J., Williams, S., Wallace, S., Ehrlich, S., et al. (2011). Global white matter abnormalities in schizophrenia: a multisite diffusion tensor imaging study. Schizophr. Bull. 37, 222-232. doi: 10.1093/ schbul/sbp088

Winkler, A. M., Ridgway, G. R., Webster, M. A., Smith, S. M., and Nichols, T. E. (2014). Permutation inference for the general linear model. Neuroimage 92, 381-397. doi: 10.1016/j.neuroimage.2014.01.060

Wu, X., Yang, W., Tong, D., Sun, J., Chen, Q., Wei, D., et al. (2015). A meta-analysis of neuroimaging studies on divergent thinking using activation likelihood estimation. Hum. Brain Mapp. 36, 2703-2718. doi: 10.1002/hbm. 22801

Zeng, T., Przysinda, E., Pfeifer, C., Arkin, C., and Loui, P. (2017). White matter connectivity reflects success in musical improvisation. bioRxiv [Preprint]. doi: $10.1101 / 218024$

Conflict of Interest: The authors declare that the research was conducted in the absence of any commercial or financial relationships that could be construed as a potential conflict of interest.

Copyright (C) 2020 Sampedro, Peña, Ibarretxe-Bilbao, Cabrera-Zubizarreta, Sánchez, Gómez-Gastiasoro, Iriarte-Yoller, Pavón and Ojeda. This is an open-access article distributed under the terms of the Creative Commons Attribution License (CC BY). The use, distribution or reproduction in other forums is permitted, provided the original author(s) and the copyright owner(s) are credited and that the original publication in this journal is cited, in accordance with accepted academic practice. No use, distribution or reproduction is permitted which does not comply with these terms. 